$$
\begin{aligned}
& \text { 震 }
\end{aligned}
$$

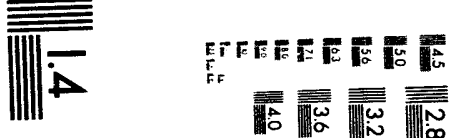

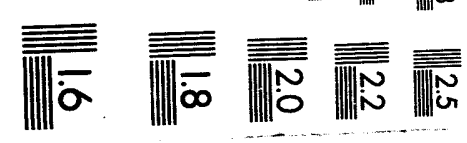



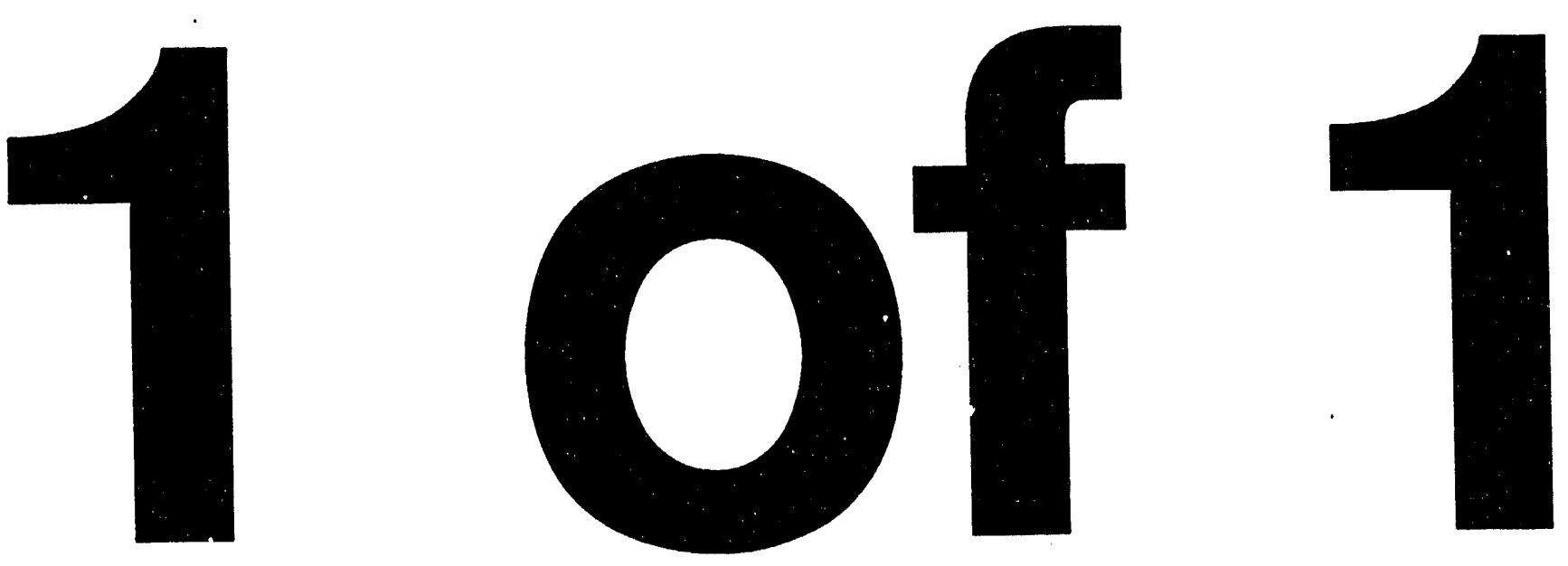


\title{
TITLE: SEARCH FOR NEUTRONS FROM DEUTERATED PALLADIUM SUBJECT TO HIGH ELECTRICAL CURRENTS
}

AUTHOR(S):

\author{
S. F. Taylor, T. N. Claytor, D. G. Tuggle, and S. E. Jones
}

SUBMITTED TO:

Fourth International Conference on Cold Fusion

Maui, Hawaii

December 6 to December 9, 1993

(Paper)

To be published in the conference proceedings

\section{DISCLAIMER}

\begin{abstract}
This report was prepared as an account of work sponsored by an agency of the United States Government. Neither the United States Gnvernment nor any agency thereof, nor any of their employees, makes any warranty, express or implied, or assumes any legal liability or responsibility for the accuracy, completeness, or usefulness of any information, apparatus, product, or process disclosed, or represents that its use would not infringe privately owned rights. Reference herein to any specific commercial product, process, or service by trade name, trademark, manufacturer, or otherwise does not necessarily constitute or imply its endorsement, recommendatir $n$, or favoring by the United States Government or any agency thereof. The views and opinions of authors expressed herein do not necessarily state or reflect those of the United States Government or any agency thereof.
\end{abstract}

By acceptance of this article, the publisher recognizes that the U.S. Government retains a nonexclusive, royalty-free license to publish or reproduce the published form of this contribution. or to allow others to do so, for U.S. Government purposes.

The Los Alamos National Laboratory requests that the publisher identify this article as work performed under the auspices of the U.S. Department of Energy.

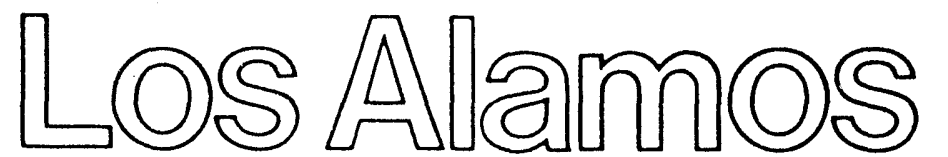

Los Alamos National Laboratory Los Alamos,New Mexico 87545 


\title{
SEARCH FOR NEUTRONS FROM DEUTERIDED PALLADIUM
}

\author{
SUBJECT TO HIGH ELECTRICAL CURRENTS \\ Stuart F. Taylor*, Thomas N. Claytor, Dale G. Tuggle
Los Alamos National Laboratory
Los Alamos, New Mexico 87545
}

\author{
Steven E. Jones \\ Department of Physics and Astronomy \\ Brigham Young University \\ Provo, Utah 84602
}

*Also with Brigham Young University

\section{Abstract}

Tritium has been detected evolving from samples of deuterided palladium wires and powders subject to pulsed high voltage at Los Alamos ${ }^{1}$. We wanted to measure whether these samples were emitting neutrons. The idea of pulsing current through the wires and powders was to drive the deuterium in and out by rapid electrical heating. With promising tritium results in hand, the experiments were prepared at Los Alamos, and then taken to BYU and run in our neutron detector located in a tunnel in Provo canyon under $35 \mathrm{~m}$ of rock and dirt overburden. The neutron detector and sample setup are described. Results including total neutron counts, time distributions, and an indication of the energy distributions are discussed. The results do not provide compelling evidence of neutron production, but are not inconsistent with earlier measurements of neutrons and tritium ${ }^{2}$. Difficulties in explaining the difference in tritium and neutron measurements are also discussed. Plans for further work are presented.

\section{Experimental Setup}

\section{Neutron Detector}

The neutron detector at BYU is referred to as a "modified Jomar detector" because it is a helium -3 tube detector designed at Los Alamos and produced by the Jomar corporation with a plastic scintillator placed in the cylindrical cavity of the Jomar detector (Figure 1). This detector is described in detail in this volume by $\&$ E. Jones et. al. ${ }^{3}$; a brief summary will be provided here.

The efficiency of the Jomar helium-3 detector alone is $34 \%$, and the efficiency of the plastic scintillator is $48 \%$, for a combined efficiency of $16 \%$. Three plastic scintillator veto counters around the detector greatly reduce the cosmic ray background; with the veto counters in place, the efficiency of the modified Jomar detector is near $15 \%$. In this tunnel, the background rate in the helium -3 detector alone is about 85 counts per hour.

To be considered valid, a detection "event" must pass software cuts, to be described. A pulse from the plastic followed by a helium-3 tube pulse within a 160 microsecond window is considered a candidate for a valid "single" neutron detection event. A single event is only 


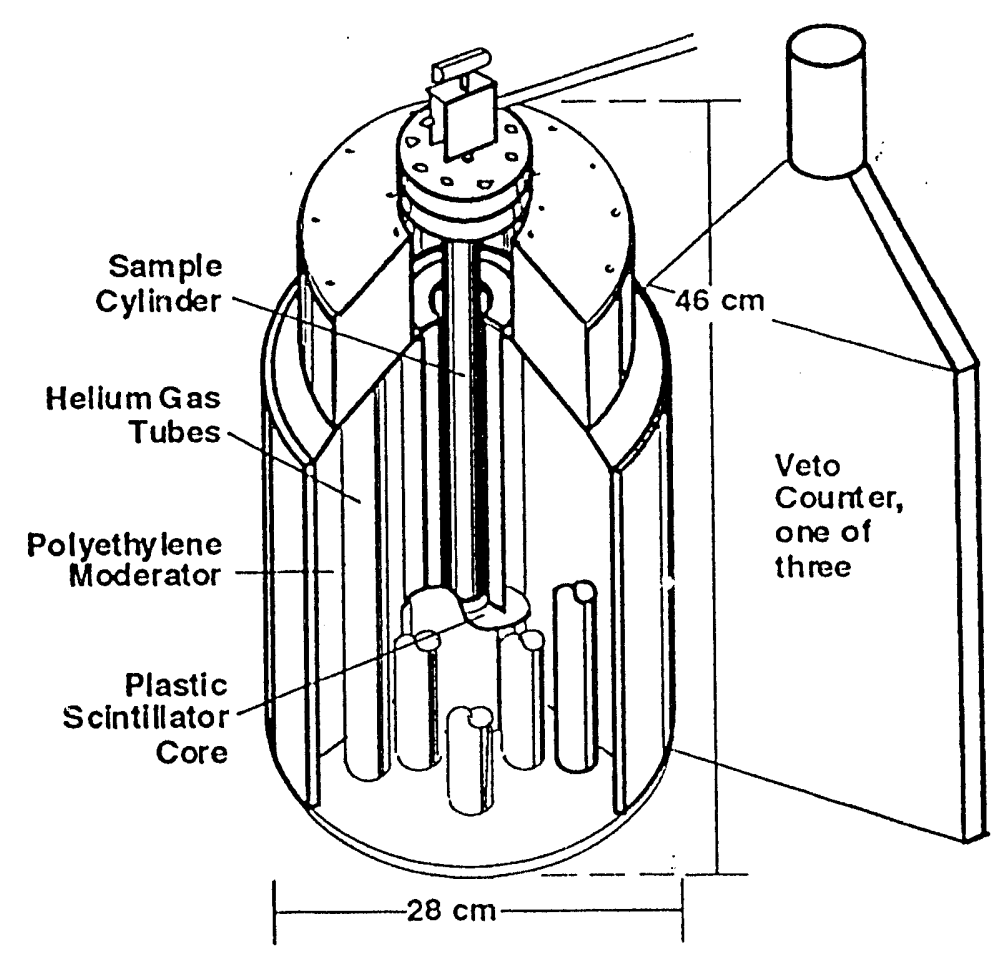

Figure 1: The BYU modified Jomar detector in a tunnel in Provo Canyon, Utah. A sample cylinder built at Los Alamos is shown inside. Not shown are two of the three large veto counters which allow removal of counts from charged particles traveling in all three directions.

considered a valid single if the area of the plastic pulse (referred to as a "start") is within certain ranges, and only if after the plastic start pulse the helium 3 tubes count a "stop" within a certain time bounds. The area of a plastic pulse is not quite a linear function of the energy the neutron deposits in the scintillator. The total energy of the neutron is not determined because not all of its energy is deposited into the scintillator. Any signal from the veto counters in these bounds invalidates the signal. The start areas are given in arbitrary units; the cut ranges are decided after a calibration study of events from neutron sources. The optimal cut accepts the most neutrons while rejecting other particles and electronic noise. A tight "cut 1 " refers to a cut that considers valid only those counts with a plastic pulse "start" area of 200 to 1500 and time bounds of 0.6 to 80 microseconds; the medium "cut 2" has an area of 200 to 1500 and time bounds of 30 to 8000 microseconds. An event is considered a "double" if the helium 3 detector detects a second neutron in the following 160 microsecond window. It is considered a "triple" if a third neutron is detected within this same time window from the second, etc. All these events of more than one neutron detection are referred to as "multiple" events. Multiple counts must be within the same time bounds, but because of their rarity, in all cuts multiples are accepted in the wider plastic area bounds of 55 to 10000 .

When combined with the plastic scintillator, the modified Jomar detector has a background count rate of 0.45 detected neutrons per hour when we require tight time correlation and narrow plastic scintillator pulse area range of cut 1 . We feel that this cut may remove too many actual neutron events; therefore, in this paper we primarily use the medium cut 2 for which we have measured a background rate of 0.7 counts per hour. Using cut 2, approximately once every 30 hours we record a multiple count. 


\section{Setup of Samples}

Earlier 1,2 , when neutrons around cells were measured in a tunnel in Los Alamos using a standard Jomar detector, it appeared that the cells were producing neutrons. Because the background neutron count is about 55 per hour in the Los Alamos "Ice House" tunnel'vs. near 0.7 per hour at the BYU detector, we prepared experiments at Los Alamos and took the experimental cells to the BYU lower background detector to measure neutrons from the cells.

The "compact" type of cell used in Los Alamos, with alternating layers of silicon and pressed palladium powder, had regularly evolved just under $0.1 \mathrm{nCi}$ per hour tritium. Based on three previous experiments that evolved high levels of tritium and also appeared to cmit neutrons at an average rate of $3 \times 10^{-9}$ neutrons per triton, our expected maximum neutron production was up to 6 source neutrons per hour, or with the BYU neutron detector's $\sim 15 \%$ efficiency, we expected to detect up to $\sim 0.9$ neutron counts per hour above background at BYU.

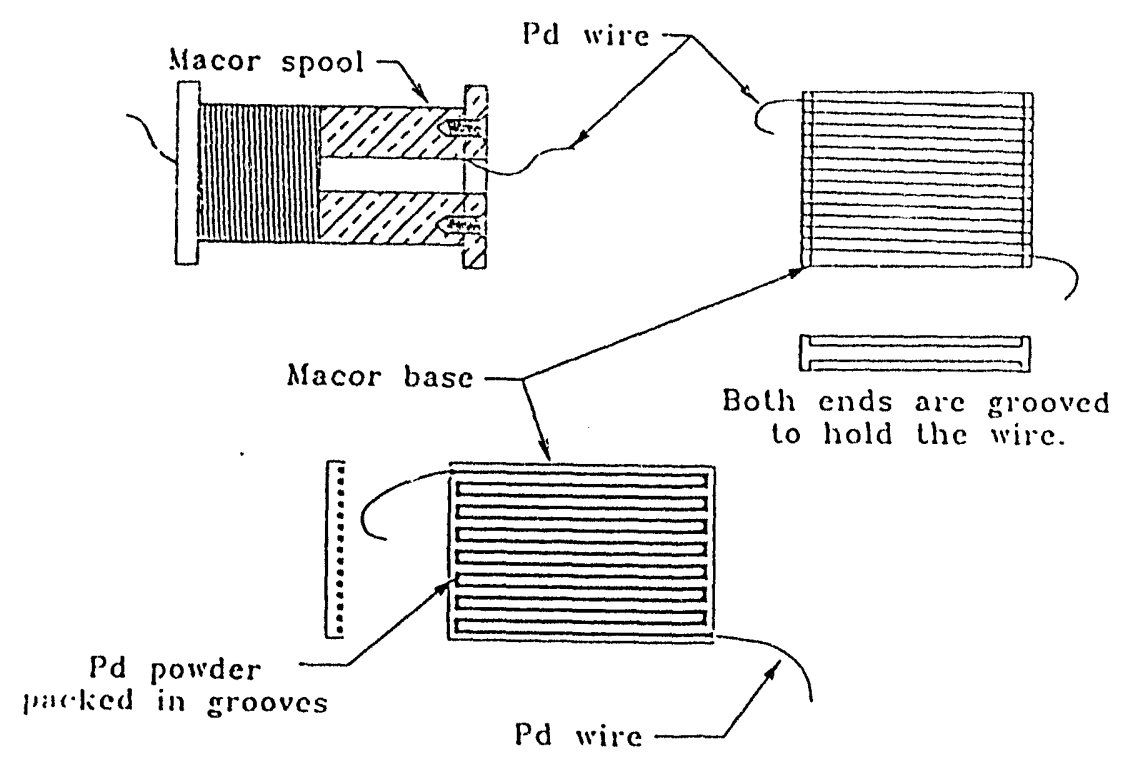

Figure 2: Schematic drawings of the three types of experimental samples used: compacted palladium powder layered with silicon, wire, and palladium powder pressed into long grooves.

A difficulty in running the compact cells is that they evolve more tritium when close to a "breakdown" voltage, that is when the voltage is nearly high enough to cause mild arcing through the layers of silicon and palladium. Operating near this limit is problematic in that if catastrophic arcing does occur, the resistance of that sample is permanently greatly decreased, and no longer shows tritium evolution. Therefore, other methods were sought that would allow faster turn around time between experiments. We therefore tried two alternate experiments where we tried to reproduce the same conditions of high current density in the palladium to see if they would evolve 
tritium and emit neutrons. The first alternate "wire" experiment was to use high purity palladium wire with a diameter on the order of $0.1 \mathrm{~mm}$. Subsequent analysis of the wires showed that the commercially available "high purity" wire in fact often contained both metallic impurities and nonmetallic impurities (i.e. carbon), most likely from the process of drawing the wire. The second alternate method was to press palladium powder into a narrow track, or "groove", and run current through the palladium in the track. These three types of samples are shown in Figure 2.

\section{BYU cell 51 and control}

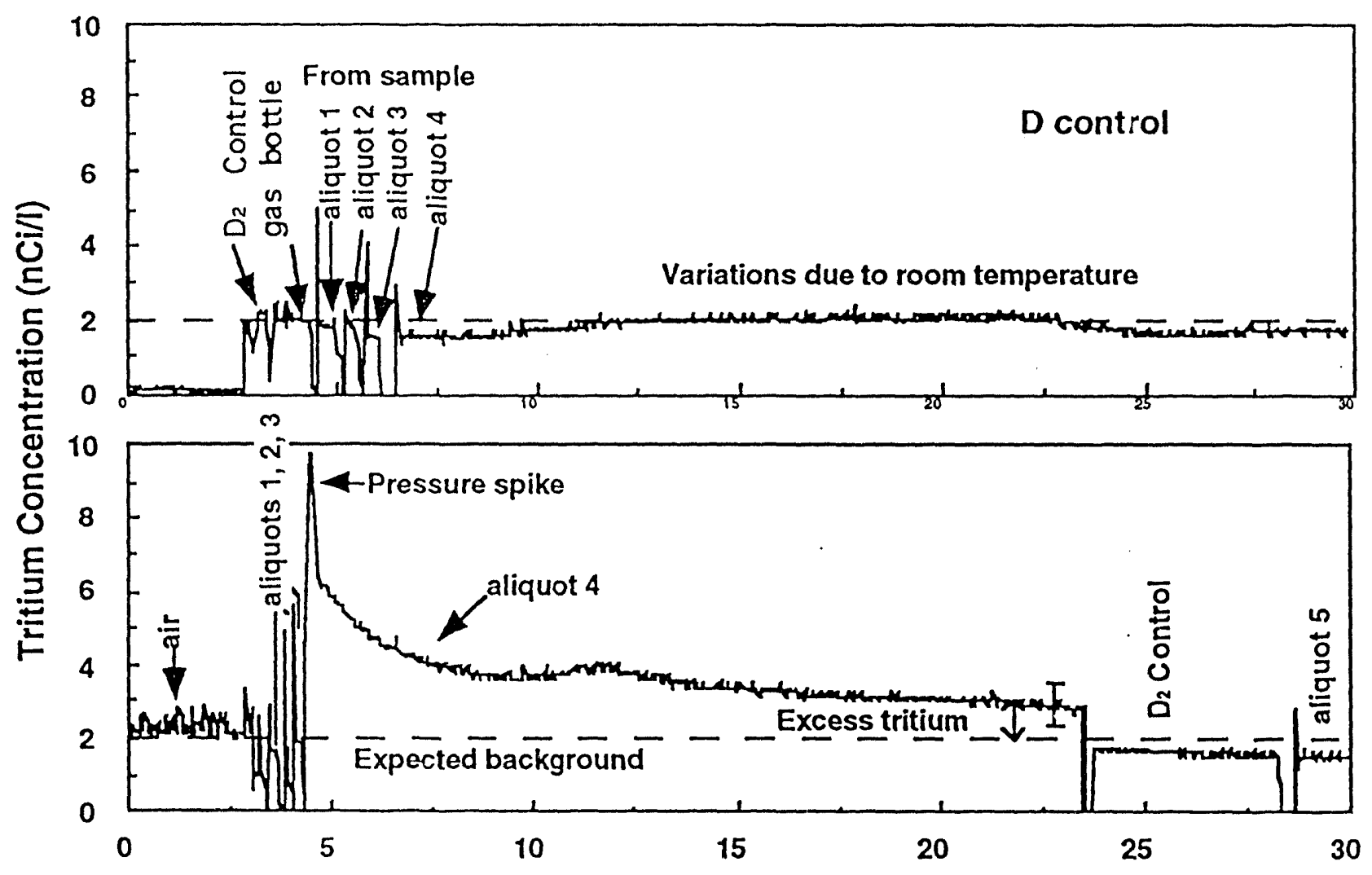

\section{Time hours}

Figure 3: Tritium measurements for compact cell 51 and a background, where the ionization current has been converted into tritium concentration. The sample gases are put into the Femtotech ionization gauge in four aliquots (shots). The background level on this ionization gauge is $2 \pm 0.2 \mathrm{nCi} /$ litre due to fixed tritium contamination in the system. Before being used in the experiments, the low tritium deuterium gas contains $2 \mathrm{nCi} /$ itre tritium (dashed line). To obtain the total amount of tritium, multiple the concentration by the total volume of the measurement system of 3 litres.

\section{Experiments Run}

The first experiment, "Wire 01", was a test of the "wire" method. Because the cell was taken to vacuum to rapidly remove deuterium absorbed in the palladium, the gas evolved was not available for tritium analysis. In this run, the neutron counts were separated into three groups of times: (1) 
When the palladium was placed in a deuterium atmosphere and therefore the deuterium was being absorbed, and current was run through the wire (an "in" run), (2) when no current was being sent through the wire, and the palladium remained in the deuterium atmosphere (a "stable" run), and (3) when current was sent through the palladium wire while it was placed under vacuum to rapidly drive deuterium out of the sample (an "out" run). Because the out runs appeared to have higher neutron outputs than the stable runs, with the in runs in between, we were encouraged to repeat the wire type of experiments. Three more wire runs were then done in a chamber prepared in Los Alamos; in these runs the wire was not put into vacuum in order that the gas could be saved for subsequent tritium analysis. Two compact experiments were run, and three groove experiments were run.

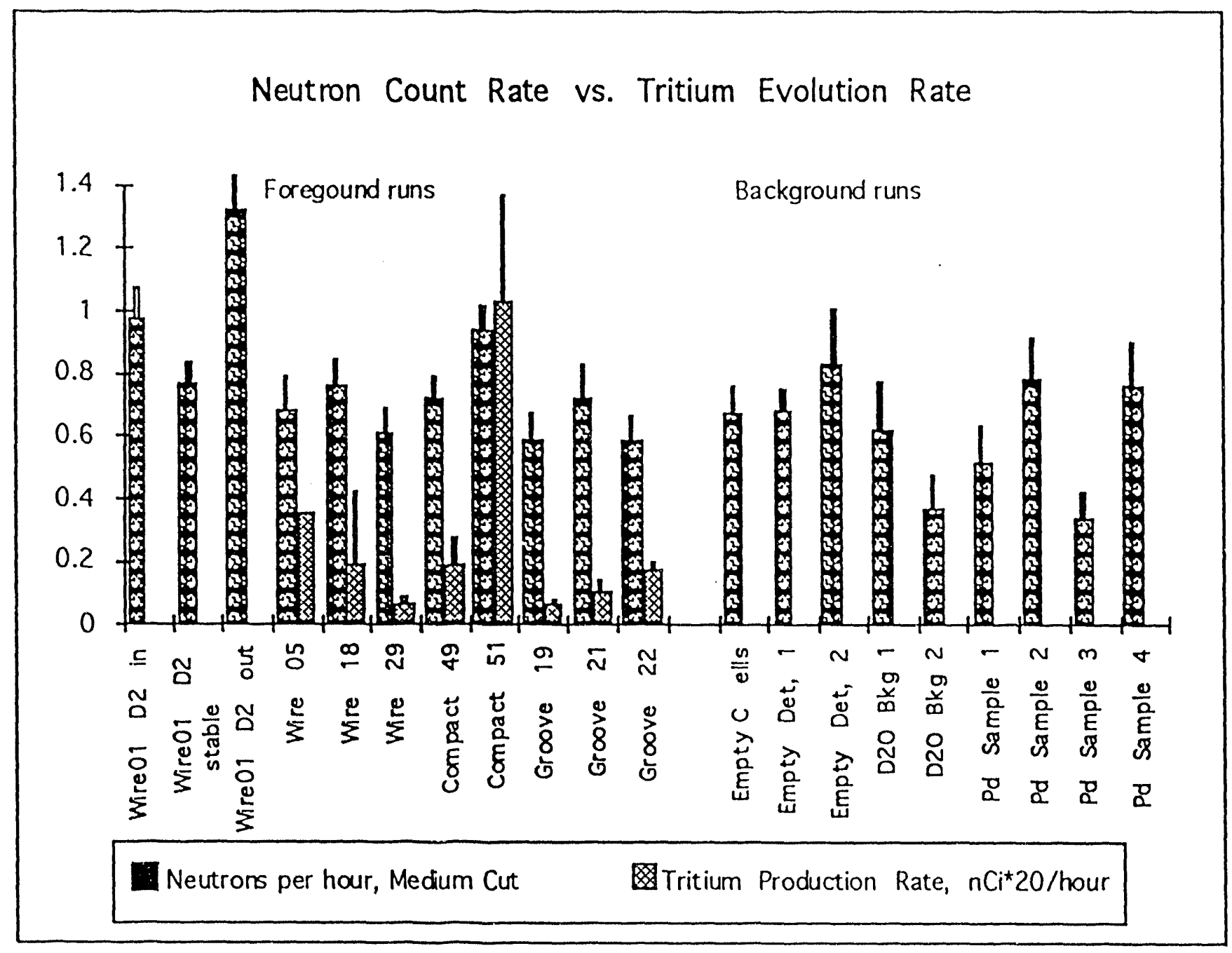

Figure 4. Neutron counts for runs done at BYU, with tritium measurements (multiplied by 20 to fit graph) shown for comparison.

\section{Total Counts Results}

Figure 3 shows the measurement of tritium for sample compact 51. This sample showed the highest tritium evolution of any sample reported here (Figure 4). The method of measurement is described in this volume 6 . We regret that the sample was not measured until the ionization current 
stabilized, as we now have a larger uncertainty in this tritium measurement than if the measurement were continued for another 10 to 20 hours.

Shown on figure 4 is a compilation of the neutrons detected during the wire run done in the BYU chamber (Wire 01), the eight experimental runs using the Los Alamos chamber (three wire runs, two compacts, and three groove runs), followed by background runs in the detector. For the runs done in the Los Alamos chamber, the quantity of tritium measured following the run the time of the run to obtain a tritium evolution rate is shown; the unit used for the tritium is nanocuries multiplied by 20 to fit on the same graph. There appears to be a higher neutron rate above background from the sample (Compact 51) from which more tritium was measured, and also a higher neutron rate in the first wire sample during the times that the deuterium is either being absorbed into or driven out from the palladium. The significance of this, however, is very uncertain due to the possibility of systematic effects; in any case, the higher neutron rates are within a few sigma of background.

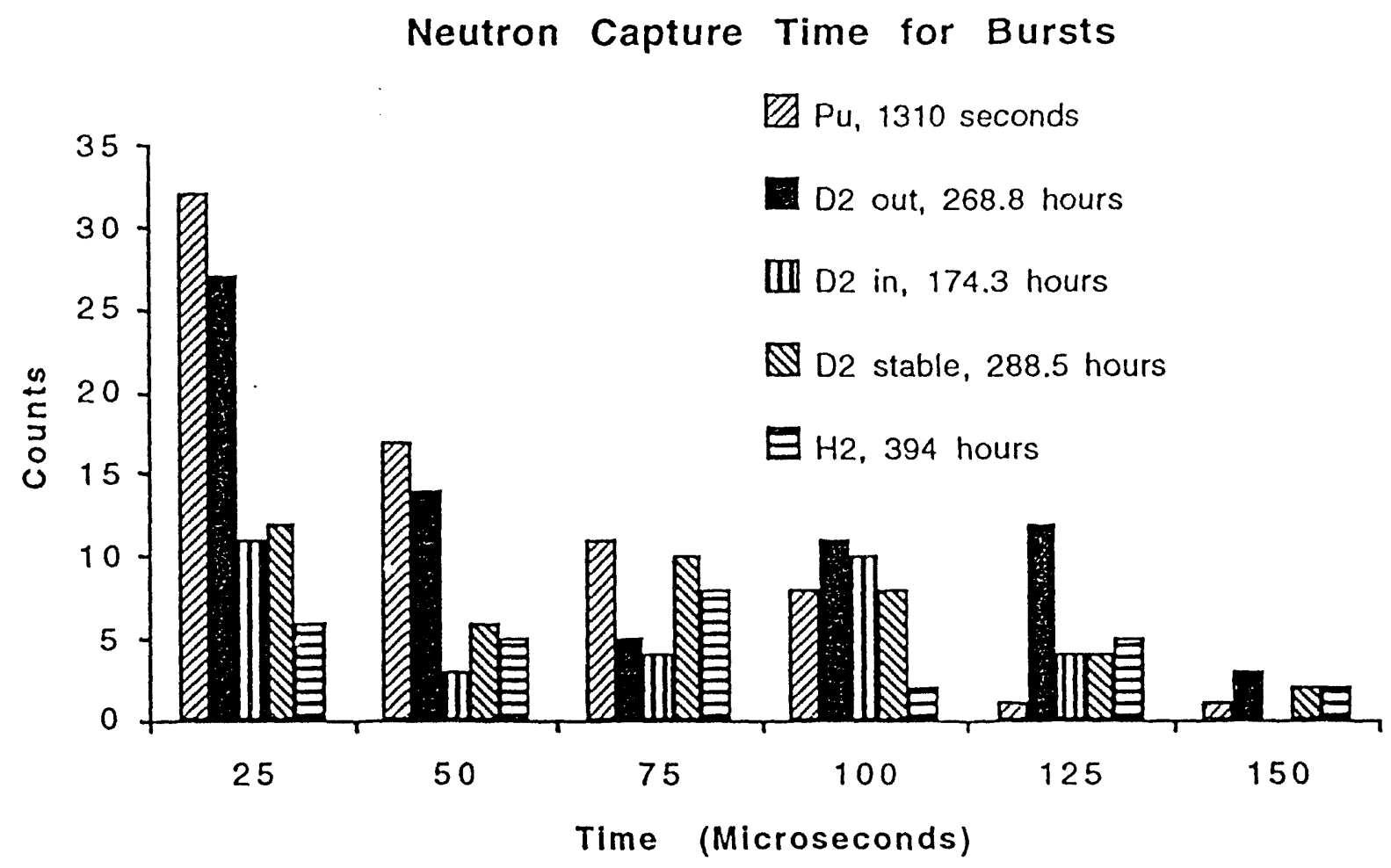

Figure 5: The time distribution of each count from multiple events shows an exponential decrease for a plutonium source where the neutrons in multiple events are "correlated" (produced simultaneously, but taking an exponentially decreasing time distribution to thermalize in the polyethelyne before being detected by the helium-3 tubes) However, in a hydrogen background where multiple events are uncorrelated this distribution randomly varies around close to the same value. The appearance of a decrease in the counts from multiples from runs in which $D_{2}$ is being driven out suggest a posible admixture of correlated counts in these runs. 
It should be noted that the background runs vary more than the one sigma error bars, which only show the statistical error associated with the number of counts. Work is underway to determine the level of systematic error, and to understand if the occasional low background runs are a result of a problem in the equipment or analysis.

\section{Time distribution of multiples}

We refer to the time from the plastic pulse to when the helium-3 counter detects the neutron as $\Delta t$.When the time distribution of $\Delta t$ are histogrammed for a plutonium source, the result is an exponential decrease with a 55 microsecond die away time. The plutonium is used for comparison because it is a good source of bursts of simultaneous neutrons -- the exponential time distribution of the detection of the neutrons is a result of the time it takes for the neutrons to thermalize in the polyethylene moderator. In other words, a 55 microsecond exponential die away time in the time distribution of the detected neutrons is indicative of the neutrons being emitted simultaneously. In contrast, when this time distribution is histogrammed for the counts from a hydrogen background, the distribution is closer to being flat, indicating less or no correlation between the plastic and the helium-3 signals. Figure 5 shows the multiple counts plotted for the runs where deuterium was being forced out of the palladium. It appears that perhaps the distribution is a mix of a flat distribution and an exponential decrease, indicating a possible source component to this signal; however, at the available level of data this conclusion is not conclusive.

\section{Energy determination}

A rough idea of the energy bounds of the neutrons that produced a start pulse in the plastic scintillator can be obtained. The total amount of scintillation of the pulse produced by the neutron, called the area of the pulse, is nearly proportional to the amount of energy deposited by the neutron in the scintillator, which is a fraction of the neutron's energy. Thus neutrons of a single energy would produce a characteristic distribution of areas. The distribution of the foreground and background runs are plotted and compared in figures 6 and 7. The small number of neutrons detected do not yet produce a very smooth distribution of the areas in either the foreground or the background; when the events in which a single neutron is detected in the helium 3 tubes are plotted it is not apparent whether there is a significant difference between the two distributions. The number of multiple events, where two neutrons are detected in the helium 3 tubes, is 18 doubles plus 1 triple in the 260 hours of foreground and 7 doubles plus one triple in the 260 hours of background. In 130 hours with a cell containing deuterium but not subject to current (called a "stable" cell) there were 3 doubles. Perhaps most significant is that all 8 multiples in the background have a plastic pulse area less than 800 , but 8 of the 19 multiples in the foreground have plastic pulse areas greater than 800 . In the stable cells, there were 2 multiples with area below 800 , and 1 count above. Greater plastic pulse areas could indicate neutrons of higher energies, or could be produced by more than one of the neutrons of a multiple event scintillating the plastic at the same time. Therefore, these multiple event results may indicate that more energetic neutrons are being emitted from foreground samples than from background, or that the neutrons in the foreground multiple events are more correlated. However, such a low number of multiple events cannot give a conclusive difference between the foreground and background. As this neutron detector is not optimized for energy spectroscopy, these results suggest more use of another BYU detector that is optimized for energy spectroscopy. A possible source of error is that the plastic scintillator is heated by the current sent through the samples in the foreground experiments; whether this results in it giving larger or spurious pulses has not yet been determined. 


\section{Neutron Multiples Counts, D2 Being, Driven Out}

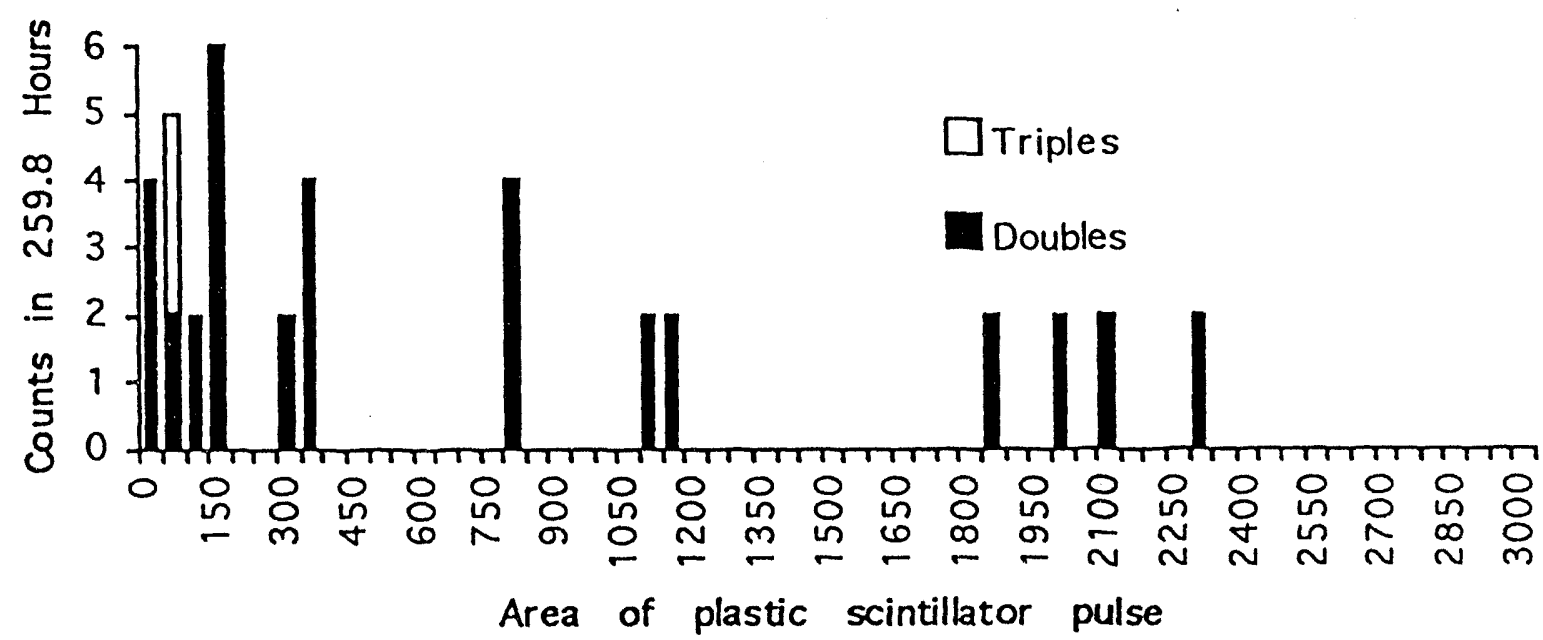

Figure 6: Number of multiples counts as a function of pulse area, plotted for 259.8 hours where deuterium was being driven out of the palladium. This can be compared with background in Figure 7.

\section{Background Multiples Counts}

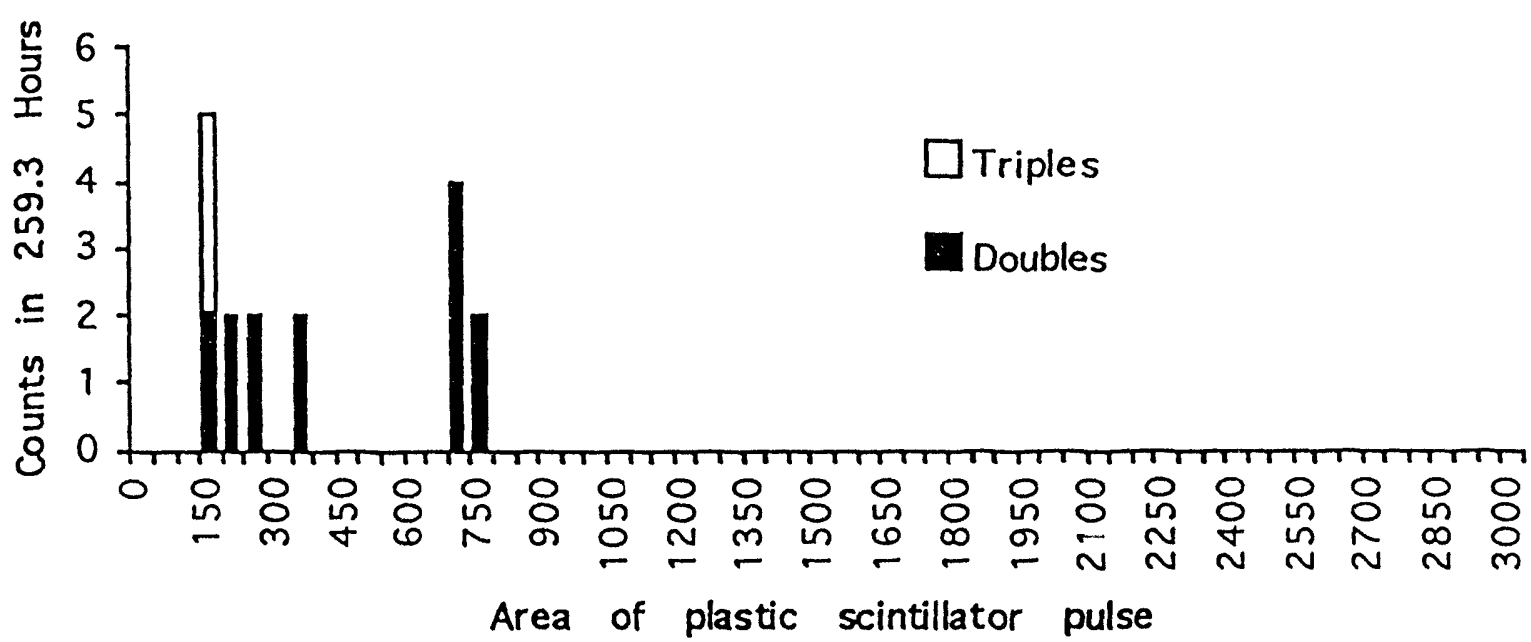

Figure 7: Number of multiple counts as a function of pulse area, plotted for 259.3 hours of background runs. 


\section{Conclusions}

The data obtained do not present a clear picture that neutrons are being produced in these experiments. What is clear is that the source neutron production rate would be much less than the roughly 1000 fusions per hour estimated as being produced in electrolytic cells run by the BYU team in $1988^{3}$; since that time, the newer detectors have much improved detection efficiency and greatly reduced background, and one would expect such a signal to now stand out clearly against the lower background. That the above indications of neutron detection are similarly close to background leads our group to be suspicious that systematic effects rather than neutrons may be producing the signals, and we are searching for these effects. There is a possibility that these data indicate a low level production of neutrons -- the evidence warrants further search, but no verified detection of neutrons is claimed at this time. It should be pointed out that the previously seen rate of $10^{-9}$ neutron/triton ${ }^{1}$ was unfortunately not well tested by the rates of tritium seen in these experiments. While tritium is being evolved fairly reproducibly in "compact" cells at Los Alamos, not every compact cell evolves significant levels of tritium (15 $\mathrm{nCi}$ or more), and the level of tritium seen in the two compact runs at BYU is consistent with marginal levels of tritium evolution. In retrospect, it may have been better to run more compact cells than wire cells, but we were encouraged by possible neutron signals detected in the first wire experiment to run more of the wire experiments. The wire experiments were also chosen because they were easier to build and were not prone to the compact cell's problem of arcing and premature failure. We conclude that the neutron rates seen were consistent with the postulated $10^{-9}$ neutron emitted per triton evolved rate, but were too close to background to be considered a confirmation.

The question of whether it is possible to have as few as only one neutron produced for every $\sim 10^{9}$ energetic tritons produced must be considered. The problem is that energetic tritium ions (tritons) produced in nuclear reactions are of the order of MeV's of energy. The reaction triton + deuteron $\rightarrow$ neutron + alpha has a resonance near $150 \mathrm{KeV}^{4}$. Any triton with an energy not less than this $150 \mathrm{KeV}$ energy resonance will produce significant numbers of "secondary" neutrons from this reaction, as the triton's energy will pass through this resonance as the triton loses energy and thermalizes. The reaction $d+d \rightarrow p+t$ produces tritons of $1 \mathrm{MeV}$; the mass differences of the reactants and products in any other energetically favorable tritium producing reaction would certainly impart much more energy to the triton than $150 \mathrm{KeV}$. It has been estimated that there should be at least $10^{-6}$ as many neutrons as tritons when the tritons lose their energy in a deuterium rich environment ${ }^{5}$, but this experiment indicates a ratio of less than $10^{-8}$. The cross sections for the $t+d \rightarrow n+\alpha$ reaction have been obtained along with code for the stopping of a triton in deuterium and palladium; this will allow a calculation of the minimum $n / t$ value for a given energy of the triton. Experimental results of this value are being sought. The lack of detection of large numbers of secondary neutrons remains the biggest challenge to the findings of tritium actually being produced in experiments where tritium had evolved.
Alternative sources of the tritium signal are being considered in this volume ${ }^{6}$. The tritium level measured in the sample Compact $51,5 \mathrm{nCi}$, corresponds to $5.4 \times 10^{9}$ atoms tritium per gram of palladium. The concentration of the tritium decay product ${ }^{3} \mathrm{He}$ in the palladium powder used in the compact cells was found to be $1.6 \times 10^{7}$ atoms ${ }^{3} \mathrm{He}$ per $\mathrm{g}$ Pd powder by Jane Potts of Los Alamos National Laboratory. Because the powder used was at least 6 years old and tritium decays into ${ }^{3} \mathrm{He}$ with a 12 year half life, the amount of tritium contamination in the palladium powder could not have been very many factors greater than this concentration of ${ }^{3} \mathrm{He}$ (unless the contamination occured shortly before the experiments and occured only in the foreground samples). If contamination was responsible for the tritium signal, this would require an anomalous capture, storage, and release of tritium. Another source of error is that some process other than tritium 
decay may produce ionization current in the Femtotech detector. This would also be unlikely as the tritium has been measured by scintillation. The possibility of false detection such as from internal leakage current is also considered in detail in reference 6 .

Gamma ray measurements of palladium samples run in experiments have been made by Jack Parker at Los Alamos, and have shown nothing above background, including from samples from experiments where tritium was detected.

\section{Further Work}

More experiments are planned at BYU with the expectation that we will obtain a run that produces more tritium, similar to more significant levels seen at Los Alamos, and thus a better test of whecher the detection ratio of $10^{-9}$ neutrons per triton is valid.

The possible energy difference of neutrons in the foreground and background deserves more attention. The Jomar/plastic scintillator neutron detector described in this paper is optimized for high efficiency and discrimination against other particles, not for energy spectrometry of the neutrons as was the neutron detector used in the initial BYU report of evidence for neutron detection from deuterided solid systems ${ }^{7}$. These experiments have not used the spectrometer due to its low efficiency relative to the modified Jomar detector; however, an energy determination would best be done in the spectrometer.

The amount of data produced by having the time information is huge, and work is progressing to improve the analysis software's ability to obtain the time and energy distributions of the helium-3 tube signals relative to the plastic scintillator signals. Also, a more flexible histogramming of the neutron signals as a function of the time during the run is being developed.

It is important that the tritium be looked for as it is being evolved in order to determine the energies of the tritons at their source. This would provide evidence that the tritium seen is actually being produced rather than merely being contamination being driven out. We plan to use a surface barrier detector to look for energetic tritons. Because the neutron to tritium detection ratio is so low, tritons of energies less than $40 \mathrm{KeV}$ should be searched for. For this, we plan on using an ion implanted surface barrier detector made for being more sensitive to lower energy tritons.

Alternative sources of the apparent neutron signal can be evaluated. The experiments do heat the detector which may generate spurious signals from causes such as cracking of the plastic scintillator. This adds another uncertainty as to whether the higher neutron signal is due to actual neutron production. We plan to reduce this problem by wrapping the cell in copper foil, and cool the foil on top. This would reduce the heat on the detector sufficiently that background runs could be done with the detector heated to the same level as the sample runs with the fear of damaging the detector.

\section{Acknowledgments}

We appreciate the help of David Beuhler, Marie Bissette, David Jones, Seth Jones, Roy Strandberg and Royce Taylor in running and analyzing the experiments. Jack Parker and David Haycock have provided us with invaluable help in measuring the gamma-ray spectra of the samples, and Jane Potts' helium-3 measurements are also greatly appreciated. We have also benefited from theoretical advice from Gerry Hale and Thurman Talley, and experimental advice from Howard Menlove. 
1. T. N. Claytor, D. G. Tuggle, S. F. Taylor. "Evolution of Tritium from Deuterided Palladium Subject to High Electrical Currents," Frontiers Science Series No. 4, Proceedings of the Third International Conference on Cold Fusion, Frontiers of Cold Fusion, H. Ikegami (Ed), Nagoya, Japan, October 1992, pp. 217-229.

Another paper that reports detection of tritium and neutrons: V. Romodanov, V. Savin, Ya. Skuratnik, Yu. Timofeev. "Nuclear Fusion in Condensed Matter," Frontiers Science Series No. 4, Proceedings of the Third International Conference on Cold Fusion, Frontiers of Cold Fusion, H. Ikegami (Ed), Nagoya, Japan, October 1992, pp. 307-319.

2. T. N. Claytor, D. G. Tuggle, H.O. Menlove, P.A. Seeger, W.R. Doty, and R.K. Rohwer. "Tritium and Neutron Measurements From Deuterated Pd-Si," AIP Conference Proceedings, Anomalous Nuclear Effects in Deuterium/Solid Systems, S.E. Jones, F. Scaramuzzi, and D. Worledge (Eds) Provo Utah, 1990, p 467.

3. Steven. E. Jones, David E. Jones, David S. Shelton, and Stuart F. Taylor. "Search For Neutron, Gamma, and X-ray Emissions From Pd/LiOD Electrolytic Cells: A Null Result," this volume.

4. G. M. Hale, R. E. Brown, N. Jarmie, "Pole Structure of the $\mathrm{J} \pi={ }^{3}{ }^{+}{ }^{+}$Resonance in $5 \mathrm{He}, "$ Physical Review Letters 59 (1987) 763.

5. J. Rafelski, personal communication.

6. D. G. Tuggle, T.N. Claytor and S.F. Taylor, "Tritium Evolution From Various Morphologies of Palladium," this volume.

7. S. E. Jones, E.P. Palmer, J.B. Czirr, D.L. Decker, G.L. Jensen, J.M. Thorne, S.F. Taylor and J. Rafelski, Nature, 338 (1989) 737-740. 


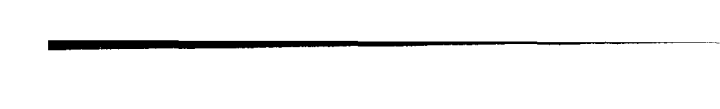

